\title{
Comunicación gubernamental y la participación de los colectivos del estado de Puebla
}

\section{Government communication and collective participation in the State of Puebla}

María Guadalupe Curro Lau ${ }^{1}$, Paola Eunice Rivera Salas ${ }^{2}$

INFORMACIÓN DEL

ARTÍCULO

Fecha de recepción: 26 de Octubre de 2017

Fecha de aceptación: 28 de Marzo de 2018

1 Maestra en Administración por el Instituto de Estudios Universitarios A.C. Docente e Investigadora de la Benemérita Universidad Autónoma de Puebla- México.

E-mail: guadalupe.curro@correo.buap.mx

2 Doctora en Educación por la Universidad Virtual Hispánica de México. Docente e Investigadora de la Benemérita Universidad Autónoma de Puebla-México.

E-mail: paola.rivera@correo.buap.mx

3 Es una inversión superior a los mil millones de dólares, estás inversiones son proyectos que impactan al entorno ambiental de las comunidades rurales de la Sierra Norte del Estado de Puebla. Que van desde oleoductos, gaseoductos, minería, plantas geotérmicas hasta empresas turísticas.

CITACIÓN: Curro Lau, M.G., \& Rivera Salas, P.E. (2018). Comunicación gubernamental y la participación de los colectivos del estado de Puebla. Podium, 33, 1-12. doi:10.31095/podium.2018.33.1

ENLACE DOI:

http://dx.doi.org/10.31095/podium.201 8.33 .1

\section{Resumen}

Este artículo tiene por objeto identificar el modelo de comunicación que se utilizó en el Estado de Puebla en el periodo 2000-2016, que incluye el análisis y evaluación del proceso de comunicación entre el gobierno y los colectivos afectados por los llamados megaproyectos ${ }^{3}$. Los resultados denotan que los colectivos utilizaron el modelo de Comunicación de Riesgos para generar una relación Gobierno-Ciudadanía basada en la negociación y el diálogo, y el modelo de Comunicación de Redes o Enjambre, donde prevalecieron mensajes a favor de la autoridad y se limitó a enviar información de acuerdo con sus intereses, cuidando su imagen ante la sociedad.

\section{Palabras Clave:}

Comunicación, gubernamental, acción colectiva, participación ciudadana, modelos de comunicación.

\section{Clasificación JEL: H70, O2.}

\begin{abstract}
This article aims to identify the communication model used in the State of Puebla during 2000-2016; it includes the analysis and evaluation of the government's communication process with groups of the so-called megaprojects. Analysis results denote that the groups that used the Risk Communication model to generate a government-citizen relationship based on negotiation and dialogue, and on the Swarm Communication Network, where messages prevailed in support of the authority were limited to sending information according to their interests, and to taking care of their image they projected to society.
\end{abstract}

\section{Keywords:}

Government, communication, collective action, citizen participation, communication models.

JEL Classification: H70, O2 


\section{Introducción}

El siguiente artículo tiene la intención de dar cuenta sobre la valoración de los modelos de comunicación que implementan los gobiernos para interactuar con los ciudadanos con los que pueden negociar situaciones de crisis, problemáticas y demandas sociales. De inicio se presenta un esbozo sobre la comunicación gubernamental, los modelos de comunicación trabajos por Elizalde y Riorda (2013) como parte del sustento teórico. Posteriormente, las autoras precisan las características de las acciones colectivas para entender cómo se integran ciertos grupos ciudadanos en pro de la defensa de causas, por ejemplo, el medio ambiente. Finalmente, se presentan algunos resultados a partir del análisis de contenido de los discursos obtenidos de cinco colectivos que participan en la defensa de sus tierras, usos y costumbres que se localizan en el Estado de Puebla, ante la inserción de proyectos con una alta inversión financiera que el propio Gobierno Estatal ha promovido y ejecutado en los últimos 5 años.

\section{La comunicación gubernamental}

Para establecer el diálogo entre el gobierno y los ciudadanos, debe plantearse (por parte del primero) una estrategia que ayude a formar un flujo de comunicación, tomando en cuenta los rasgos socioculturales y coyunturales que existen entre los diferentes grupos sociales. Al establecer este diálogo se permitirá satisfacer las necesidades que se presentan, y generará participación ciudadana y una buena imagen del mismo. De esta forma surge la comunicación gubernamental, que se encarga de mantener un control sobre la ciudadanía a la que comunica las actividades que un gobierno lleva a cabo. Si las necesidades no son cubiertas por medio de estas actividades, se genera una relación tensa entre el gobierno y los ciudadanos que sólo puede ser evitada si se establecen políticas públicas adecuadas que busquen el desarrollo óptimo de las personas (Elizalde y Riorda, 2013).

Por ello, la comunicación es fundamental al ser utilizada como una herramienta para mejorar el proceso, lo que conlleva a la mejora de la imagen y de las tareas de carácter prioritario. Riorda (2011), menciona:

La comunicación gubernamental juega un papel clave en la construcción de una determinada cultura política. Se cultiva desde ella el rol deseado de los atributos de la ciudadanía y, complementariamente, se crean condiciones materiales y no materiales para sostener esa ciudadanía, a través del desarrollo de símbolos y mitos que configuran elementos de identidad. Ese propósito es también un objetivo (párr. 16).

Cuando se presenta la participación es la sociedad quien promueve y avala la toma de decisiones, para desarrollar políticas públicas adecuadas a sus necesidades. Por ello la ciudadanía debe mantener un diálogo constante, que facilite la relación entre el gobierno y la sociedad. 


\section{Modelo de comunicación}

Se define como modelo al instrumento cuya finalidad es ver la realidad con una mirada reduccionista, es decir, solo retoma ciertos elementos de un fenómeno y los pone en manifiesto para poder analizarlos (Rodrigo, 2014). Elizalde y Riorda (2013) han propuesto ocho modelos acerca de la comunicación de la realidad. La elección de estos modelos son apropiados para buscar y lograr consenso y legitimidad; que aunque se entiende que existen temas ya establecidos o impuestos de manera constitucional, la agenda pública busca mantener un equilibrio social, entre otras cosas. Estos modelos proponen en términos de comunicación, que esta sea entendida más allá de los medios masivos de comunicación, esto es, desde una dimensión simbólica, que puede permitir conocer si la comunicación ejercida tradicionalmente o con mayor frecuencia por los gobiernos conllevan a una gestión eficiente, sostenible y con procesos de retroalimentación y participación social y que apunten hacia la construcción de significados compartidos y que permitan la acción de todos los actores. Conviene recordar en este punto que las instituciones públicas justifican su existencia a través de la dualidad de sus papeles: autoridad y servicio (Cancelo, 2007). Sin embargo, para cumplirlos de manera eficiente requieren de una legitimación social, la participación y apoyo de sus públicos o interlocutores sociales.

Molina, Curro, y Carrillo (2016) describen la dimensión de la comunicación gubernamental a partir de varios aspectos como son: a) que es un proceso que conlleva tareas o actividades realizadas de manera diaria o continua, tangible o intangiblemente; b) que maneja información especializada; c) que utiliza canales o materiales físicos para transmitir un mensaje; d) que sigue un proceso dinámico y cambiante acorde a las situaciones y contextos en los que se lleva a cabo; e) que los interlocutores tienen papeles y funciones específicas; f) que tiene como finalidad influir sobre la opinión pública general, hacer más eficiente la gestión pública, establecer lazos comunicativos estables entre las instituciones y sus públicos (diálogo), aliviar la tensión social, mantener o construir significados compartidos y generar credibilidad y confianza en los ciudadanos; y g) que es histórica. Los procesos institucionales deben abrir, crear y promover espacios para el encuentro comunicacional entre los entes organizacionales y sus públicos, en donde se aplique la actividad dialógica, la cual sería el resultado de una puesta en común tanto desde la organización como desde los ciudadanos, con el objetivo de posibilitar el cambio o modificaciones de comportamientos o actitudes del otro.

Los Modelos de Comunicación Gubernamental, explicados por Elizalde y Riorda (2013), se retoman para explicar el proceso de comunicación como una estrategia para llegar al público de manera participativa, los cuales son:

- Modelo de difusión y política de "enjambre" (swarming): propone la construcción y envío de mensajes que son de interés público para la sociedad y por medio de diversos canales.

- Modelo de identificación o identidad: la credibilidad del emisor es de vital importancia porque de esta forma es 
reconocible por la sociedad y supone una fuente confiable sin el mal uso de la información.

- Modelo de relaciones con agentes: la negociación con grupos de presión y afines a las ideas del gobierno como medida para evitar una crisis.

- Modelo de comunicación de marketing (marketing communication): al segregar a la audiencia en tipos, se puede conocer la forma en la que las ideas presentadas por el gobierno pueden ser aceptadas por la ciudadanía.

- Modelo comunicación de riesgo (risk communication): el diálogo es visto como herramienta clave para conocer la percepción que tiene la sociedad sobre el riesgo.

\section{Comunicación participativa}

Castells (2012) afirma que los movimientos sociales dependen de la existencia de mecanismos de comunicación. Las características de los procesos de comunicación ente los individuos que están inmersos en los movimientos determinan su forma de organización. La comunicación participativa es entendida como un modo de comunicación alternativa que promueve la democracia y la participación ciudadana. Este tipo de comunicación es comúnmente utilizada para temas de desarrollo, cambio social, paz y salud. Esta dinámica genera una discusión de temas de interés colectiva sobre prácticas extensionistas y difusionistas con el fin de fomentar el diálogo y acabar con ámbitos jerárquicos que estas prácticas proponen. Así, se busca el cambio social y la apropiación de los procesos comunicacionales por medio de la participación (Del Valle Rojas, 2007).
Una tarea que ha tomado relevancia para las Organizaciones No Gubernamentales (ONG) y el sector académico es la comunicación participativa, que debe alejarse del modelo unidireccional y vertical que impide el reparto equitativo del diálogo en el proceso de toma de decisiones y reduce la posibilidad de participación al no existir forma alguna de integrarse. Debe enfocarse en el factor humano para la apropiación de los procesos comunicacionales y la viabilidad de los procesos de desarrollo. Por ejemplo, el modelo de marketing social, en el modelo de flujo de etapa única que consiste en distribuir el mensaje por medios de comunicación (televisión, radio, revistas, periódicos) que lo llevan directamente al individuo; es pues que gracias a la proliferación tecnológica (no redes sociales) existe la posibilidad de difundir mensajes con mayor alcance. Aunque, este mensaje puede ser ajeno al contexto y como existe poca o nula retroalimentación, puede o no cumplirse el objetivo. La comunicación participativa, en cambio, busca el reparto equitativo mediante la comunicación horizontal para aumentar la concientización sobre aspectos individuales y colectivos (Barranquero y Sáez, 2012).

La participación se ve enfocada en las personas, pues al involucrarse en el proceso y formar parte activa de él pueden influir en el desarrollo de políticas y en la adopción de políticas públicas apegadas a necesidades reales que presente la comunidad. También, se busca promover actitudes y prácticas, cuyo fin sea generar un beneficio tomando en cuenta la realidad social y la cultura de la comunidad (Richter-Morales, 2014). 


\section{Acción colectiva y colectivos}

La inserción del neoliberalismo tiene como repercusión el deterioro en las condiciones de vida de la población, dando como resultado la creación de algunos movimientos populares, cuyo fin es contrarrestar dichas deficiencias. Estos escenarios pueden ser locales, nacionales o globales donde las redes de interacción son cada vez más complejas, presentando la diversidad y la complejidad de la vida social (Bokser y Salas-Porras, 1999).

La acción colectiva surge como la unión de actores sociales enfocada en el logro de fines específicos que atañen tanto intereses individuales como colectivos y donde se busca el cambio de políticas públicas cuya visión es reduccionista. Los integrantes, al compartir características en común, construyen las identidades colectivas que emergen, entonces, en diversos escenarios y ante un contexto global (Villaveces-Niño, 2009). La acción colectiva aparece cuando los ciudadanos corrientes, en alianza con algunos otros ciudadanos influyentes, unen sus fuerzas para enfrentar a las élites, el gobierno o cualquier actor que represente su antagónico social (Richter-Morales, 2014).

En la acción colectiva se promueve la reflexión, proponiendo criterios morales para valorar situaciones de exclusión y dominación. También se busca erradicar o modificar estereotipos, promover el cambio en las relaciones sociales y en el concepto de ciudadanía al requerir la participación de dos o más individuos que puede presentarse de forma espontánea o bajo la intervención de una institución centralizada. Esta participación requiere de una conexión entre los individuos respecto a espacio y tiempo, y que se vincule a la resolución de un propósito previamente establecido (Delgado-Salazar, 2011).

La solidaridad se ve ligada a los miembros en el sentido de que debe de estar presente durante el proceso de interacción. Si no hay una visión común, -como una injusticia-, no se puede legitimar la acción; por tanto, tampoco se puede construir una identidad. La decisión del individuo sobre si debe participar o no, se ve fundamentada en la percepción que tiene sobre el otro (Melucci, 1994, citado en Delgado-Salazar, 2011; Villaveces-Niño, 2009).

La acción colectiva puede ser entendida desde distintas teorías. Así, por ejemplo, Kahan (2002 citado en Villaveces-Niño, 2009) asegura que la decisión que toma el individuo de cooperar o no, estaría determinada por la percepción que tiene de su contraparte y de la consistencia en la cooperación de otros miembros del colectivo. Asimismo, la no-cooperación puede generar en los individuos conductas guiadas por la venganza y el resentimiento. En la tabla 1 se resumen algunos de estos acercamientos con dicho fenómeno, que buscan entender este tipo de movilización de distintos actores por un objetivo concreto.

Es importante señalar que las acciones colectivas son la base de los movimientos sociales. Estos conglomerados no sólo se limitan a protestar; muchos de ellos generan organizaciones, construyen ideologías, movilizan electores y se 
Tabla 1.

Teorías sobre la Acción Colectiva

\begin{tabular}{ll}
\hline Nombre de la Teoría & \\
\hline Teoría Olsoniana & $\begin{array}{l}\text { El individuo no participará en acciones colectivas a no ser que implique un incenti- } \\
\text { vo realmente relevante para él. } \\
\text { Representa los resultados de la no cooperación, la cooperación o la cooperación } \\
\text { unilateral. Los individuos se enfrentan a dilemas sociales al tomar la decisión de } \\
\text { formar o no parte del colectivo. }\end{array}$ \\
Luego de la gallina & $\begin{array}{l}\text { que todos los individuos tienen intereses diferentes. } \\
\text { Cada individuo coopera pues da por supuesto que los demás cooperarán; hay } \\
\text { consenso en la dirección que debe tomar el colectivo. } \\
\text { Juego de seguridad }\end{array}$ \\
Juego privilegiado & $\begin{array}{l}\text { Plantea que la acción colectiva será exitosa, ya que un miembro determinado dentro } \\
\text { del grupo tiene incentivos suficientes para suministrar el bien colectivo. }\end{array}$ \\
Dilema del altruista & $\begin{array}{l}\text { Bajo esta perspectiva, los individuos del colectivo son altruistas, y todos cooperan } \\
\text { porque es lo que los otros esperan que haga. }\end{array}$ \\
Reglas heurísticas & $\begin{array}{l}\text { Asume reglas de cooperación dentro del colectivo, dónde los individuos continua- } \\
\text { rán colaborando a mayor nivel, si observan que los demás lo hacen. }\end{array}$
\end{tabular}

Fuente: Miller Moya (2004) y Villaveces-Niño (2009).

forman como ciudadanos

(Richter-Morales, 2014).

\section{Acción colectiva, participación ciudadana y la comunicación gubernamental}

Los colectivos son concebidos como sistemas de acción que se construyen gracias a la comunicación, la negociación y el conflicto, esto porque se propone la inserción de prácticas sociales y culturales en contra de las deficiencias presentes dentro de la sociedad. Su nivel de acción se ve reflejado en el grado que la problemática presenta - de lo local a lo internacional-, toma diversas formas de lucha que va desde huelgas hasta la publicación de memorándums (Villaveces-Niño, 2009).

Su impacto se ve reflejado en diversos indicadores como lo son el nivel de acción, la capacidad de movilización, de coordinación y de lograr alianzas. La influencia que estos movimientos tienen ha ocasionado la reducción del poder por parte del Estado para poder combatirlos, pues no sólo se tiene que enfrentar a la tarea de gobernar con organismos internacionales públicos, no gubernamentales, privados y cívicos; sino que, al generarse nuevas formas de participación política, tiene que enfrentarse a las nuevas formas de reagrupamiento de la sociedad civil y a la construcción y reconstrucción de la ciudadanía (Bokser y Salas-Porras, 1999).

Por otro lado, la participación se define como el esfuerzo organizado para aumentar el control de los recursos y de las instituciones reguladoras de partes de grupos y movimientos hasta ahora excluidos de tal controversia. El trabajo 
participativo ha crecido porque más y más organizaciones están encontrando que pueden obtener resultados significativamente mejores utilizando métodos participativos, en lugar de la elaboración de políticas tradicionales aplicadas por las administraciones gubernamentales. No obstante, en ocasiones la participación sólo ocurre porque es necesario, más que por una iniciativa aleatoria. Las organizaciones están encontrando que esta modalidad encaja muy bien con la idea de permitir al gobierno llegar directamente a los consumidores, los ciudadanos y las comunidades que tienen a su cargo, para tener servicios públicos más eficaces y eficientes (Involve, 2011).

Las acciones colectivas pueden ser un verdadero abanico de posibilidades de manifestación sobre las inconformidades de la ciudadanía. Para Hamlichi (2008) los colectivos toman tanto diversas formas de lucha, como distintos niveles de confrontación. En función de las actividades que llevan a cabo cada movimiento se puede hablar de marchas, concentraciones, caravanas, huelgas, o publicación de memorándums. No obstante, se pueden dar otro tipo de expresiones creativas cómo la vestimenta, adoptar señales o signos, e incluso a través del arte (Richter-Morales, 2014).

Hamlichi (2008) ha señalado que el impacto transformador y reivindicativo de los grupos colectivos puede ser valorado según indicadores como el nivel de acción, la capacidad de movilización, de coordinación y de lograr alianzas; el impacto social, la influencia reivindicativa, política o transformadora que alcancen. No obstante, la acción colectiva no siempre tiene capacidad de incidir en las políticas públicas; su impacto está relacionado con los actores que integran las organizaciones, el apoyo de la opinión pública, el clima institucional, entre otros (Villaveces-Niño, 2009).

Los colectivos han significado un gran reto para la autoridad estatal. Diversos autores coinciden en que, el Estado ha perdido eficacia para reglamentar y aplicar sanciones a las Organizaciones No Gubernamentales; quiénes a su vez han integrado comunidades e identidades más allá de las fronteras nacionales. Sin duda, este tipo de conglomeraros replantean los nexos que se dan entre los ciudadanos desde lo local, nacional y global (Bokser y Salas-Porras, 1999; Hamlichi, 2008)

Colombo (2012) señala que la participación ciudadana tiene distintos niveles no excluyentes y graduales. Estos niveles incluyen: 1) Información, 2) Comunicación, 3) Consulta, 4) Deliberación y 5) Participación en la toma de decisiones. Como se observa, es necesaria la gestión adecuada de la comunicación para alcanzar niveles de participación que cubran las aspiraciones por las que se crearon los colectivos.

\section{Metodología}

La metodología usada es de tipo cualitativa, diagnóstica, de corte transversal y a partir de un muestreo de conveniencia (Ruiz, 2012). Los modelos tomados como referentes para ver si eran aplicados fueron: el modelo de difusión y política de "enjambre" (swarming), el 
modelo de identificación o identidad, el modelo de relaciones con agentes: la negociación con grupos de presión y afines a las ideas del gobierno como medida para evitar una crisis, el modelo de comunicación de marketing (marketing communication), y el modelo comunicación de riesgo (risk communication). Se realizó una transcripción de las participaciones de los grupos sociales, llamados Colectivos. En la figura 1 se presenta la descripción de las características de los grupos considerados, que están compuestos por habitantes de diferentes poblaciones o zonas de la Sierra Norte del Estado de Puebla y se han unido con el objetivo de contrarrestar la invasión y los daños que los megaproyectos están generando en sus comunidades.

El Cuerpo Académico "Comunicación y Sociedad" de la Facultad de Ciencias de la Comunicación de la Benemérita Universidad Autónoma de Puebla, recabó los mensajes de los colectivos en el Coloquio "Comunicación Ambiental", realizado en el segundo semestre de 2017. Con esta información, se elaboró un análisis de los discursos emitidos por los colectivos, el cual se concentró en varias matrices para procesar los mensajes y una lista de cotejo diseñada en función de las variables de estudio: Comunicación Gubernamental, Colectivos, 
Comunicación Participativa. A partir de un enfoque empírico, se distinguió la presencia de dimensiones e indicadores de cada una de las variables y se procedió a un cruce teórico con los dos enfoques usados.

\section{Resultados y discusión}

\section{Interpretación Comunicación Gubernamental}

En el análisis de las participaciones de los colectivos o grupos sociales en pro del cuidado y defensa del medio ambiente, específicamente de las regiones de donde provienen (Sierra norte del Estado de Puebla), se realizó un cruce de información dentro de un cuadro de clasificación en donde se encuentran las características de los Modelos de Comunicación Gubernamental que pueden ser usados para la temática ambiental. Así entonces el cruce entre la información que los colectivos dieron sobre cómo se comunican, tanto con el gobierno como con la sociedad en general, indicaron que el primer modelo, Swarming, intentó explicar la forma en que se pueden establecer flujos de comunicación que permitieran distribuir $\mathrm{y}$ producir información respecto a la imagen del gobierno y su relación con medios de comunicación y líderes de opinión. Esta información puede ser consumible por toda la sociedad. En el análisis del modelo se encontró que la gran mayoría de los discursos cumplían con un carácter de difusión, estableciendo líneas de comunicación por las que fluía información respecto a la imagen que la opinión pública tenía del gobierno como lo menciona Elizalde y Riorda (2013). De lo que carecían estos discursos era de impacto en los medios de comunicación por lo que perdían fuerza. Además, sobresale que los colectivos analizados no presentan alguna característica del uso de este modelo.

El segundo modelo de Identificación o Identidad, intenta unificar criterios y atributos básicos del emisor con el fin de identificarlo. En el análisis se pudo apreciar que la mayoría de los discursos buscaban unificar criterios y dejar en claro quién era el responsable de los mensajes, siempre buscando comprensión de comunicación. En el caso de los Colectivos, era su búsqueda de reconocimiento del trabajo que estaban realizando para la defensa de sus tierras y del medio ambiente. Por parte del gobierno, también querían dejar claro que "ellos" eran los que realizaban estrategias de apoyo para el cuidado del medio ambiente. Los discursos iban en función de que cada actor defendiera su imagen, es decir, pretendían apelar a la credibilidad en torno al emisor, usando a los medios de comunicación como un canal para posicionarse. En contraste con Elizalde y Riorda (2013) si bien se busca la credibilidad del emisor, no se expone información que incluya todas las perspectivas sobre el gobierno del Estado de Puebla, por lo que es una exposición parcial de este agente.

En el análisis del tercer modelo, se encontró que el discurso gubernamental se centraba en el Modelo de Marketing Communication, es decir, su objetivo era informar, persuadir o motivar cambios de comportamiento. Ninguno de los colectivos pensó en realizar un posicionamiento como agente de cambio. Sólo uno de ellos cubrió una de las características de este modelo que señalan Elizalde y Riorda (2013) que fue el uso de los medios de comunicación colectiva para propiciar aceptación de ideas sociales, y posicionarse en la mente de la ciudadanía. Sin embargo, esto no significaba que desearan 
realmente un cambio de comportamiento social; en general, estaban más enfocados en defender sus tierras y en ellos, y en que el Gobierno los tomara en cuenta, que el cambiar todo el contexto.

Con respecto al modelo de Relaciones con Agentes, es un proceso de negociación a través del diálogo en donde se establecen acuerdos que beneficien la imagen del gobierno; en este sentido la aportación realizada por el gobierno no determina que use este modelo, solo explica que están apoyando a las comunidades, al Estado, y a proteger al medio ambiente, pero no determina si han hablado con los actores de esas comunidades. Todo lo contrario, los colectivos sí requerían y necesitaban estar en contacto con el gobierno, de hecho, solicitaron que este les preste mayor atención y dialogue con ellos para evitar crisis tanto en sus comunidades, el medio ambiente y en la relación con el Gobierno.

El modelo de Risk Communication, es usado para establecer información sobre los riesgos, sobre todo de desastres naturales, ya sea que exalten o minimicen el efecto de estos, dependiendo de los intereses del gobierno. Así, en los discursos, se pudo notar que era el que estaban usando en sus mensajes para cuidar a la ciudadanía de algún desastre natural, con respecto a la situación que se estaba viviendo en la Sierra Norte de Puebla. Aunque los colectivos, desde el punto de vista teórico, se apegan más por sus características a este modelo, en este caso solo presentaron las siguientes características: 1) Tomar riesgos en cuestión del cuidado de sus comunidades y a través del diálogo llegar a una negociación sobre la situación, y 2) Solicitar que se les informara con claridad y estar cercanos a los medios y al gobierno para tener dicha información de primera mano.

En resumen, se puede dar cuenta que el modelo que más características aplicables tenía por los colectivos, según el planteamiento de Elizalde y Riorda (2013), fue el de Risk Communication, debido al diálogo y la negociación que se pudo establecer entre el Gobierno y los grupos sociales. No obstante, el Gobierno definitivamente cuidó más la imagen que daba hacia toda la sociedad, la relación con los medios de comunicación era muy importante para emitir información que lo mantuviera vigente y buscaba el apoyo de líderes de opinión, es decir, el modelo más usado por el gobierno fue el Swarming.

\section{Interpretación Acción Colectiva}

Con respecto a la forma en que se presentaba la acción colectiva dentro de las organizaciones. Se observó que todos los colectivos analizados presentaron las siguientes características:

a. Manifestaron un interés común que los integró como agrupación que menciona necesario Villaveces-Niño (2009). También, se percibió un sentido de solidaridad y confianza entre sus miembros. No obstante, se observó que no existía una identidad colectiva en ninguna de las agrupaciones, como lo señalan Bokser y Salas-Porras (1999).

b. Estos grupos mostraron claramente que su objetivo era atender una problemática social de tipo ambiental. Adicionalmente, las diversas actividades que habían venido realizando, se enfocaron en resolver la 
problemática detectada que afectaba sus intereses como ciudadanos. Dichas actividades también constituían una respuesta a la posición ortodoxa a las políticas públicas centradas en el Estado. En este sentido, se cumple con lo expuesto por Richter-Morales (2014) en cuanto al objetivo que los grupos de ciudadanos persiguen.

c. Igualmente, con base al discurso articulado destacó que estos sujetos se concebían como espacios educativos que coadyuvaban a la construcción de nuevas realidades sociales.

Se reportaron solo algunas divergencias. En cuanto a la expectativa de éxito, sólo uno de los cuatro colectivos manifestó su expectativa de obtener los resultados esperados por su colectivo. Asimismo, tres de los cuatro colectivos se cuestionaron seriamente sobre el concepto de ciudadanía y participación ciudadana vigentes en la sociedad.

\section{Conclusiones}

De acuerdo con el objetivo de esta investigación, se identificó que el modelo de Comunicación Gubernamental que usó el Gobierno fue el modelo Swarming, donde la información que emitían siempre era a favor del gobierno y sólo se limitaron a enviar información de acuerdo a sus intereses, siempre cuidando su imagen ante la sociedad. En este modelo, el Gobierno, únicamente, protegió su imagen y sus intereses. Con respecto a la protección del Medio Ambiente que estaban solicitando los colectivos, no hubo una resolución favorable a ellos, debido a la falta de consenso tanto en el diálogo como en el modelo de comunicación gubernamental usado. Si bien este modelo había sido aplicado por el Estado, era preciso generar flujos de comunicación bidireccionales que llevaran a la atención de los reclamos de los colectivos y que ayudara a mantener la buena imagen del gobierno.

En cambio, los colectivos usaron el modelo de Risk Communication, el cual funcionó para generar una relación Gobierno-Ciudadanía basada en la negociación y el diálogo, con un trasfondo de protección en donde ambos actores estaban "protegiendo" sus intereses, por lo tanto, los colectivos lo usaron para relacionarse con el Gobierno por su carácter de prevención, con el fin de tener una participación pro activa estableciendo canales de comunicación con base en la negociación y el diálogo. Pero al parecer carecieron de información sobre cómo funcionaba este modelo en específico, por lo que se pudo observar que lo realizaron de manera empírica y fueron aprendiendo de acuerdo a su experiencia. Cruzando con los resultados de la variable de Acción Colectiva, en donde, se apreció que los colectivos cuestionaron seriamente la forma en que el gobierno tomó decisiones en cuanto a las problemáticas ambientales, se destacó, que hubo una comunicación más de corte vertical y poco incluyente. Por lo que se pudo inferir, que estos grupos hubiesen decidido integrarse como organizaciones independientes que manifestaban su inconformidad ante las medidas estatales. Asimismo, el tipo de participación que llevaron a cabo fue activa y se manifestó en la organización 
que tenían entre sus miembros, además de que echaron a andar diferentes programas comunitarios demostrando trabajo en equipo, y considerando las necesidades de los habitantes. Sus programas cumplieron con las etapas de planeación, acción y evaluación; fueron incluyentes y proactivos en el sentido de que colaboraron en la elaboración de los planes de desarrollo y ordenamiento de sus comunidades.

\section{Referencias}

Barranquero, A., y Sáez, B., Ch. (2012). Teoría crítica de la comunicación alternativa para el cambio social: el legado de Paulo Freire y Antonio Gramsci en el diálogo norte-sur. Razón y Palabra, 16, (80), 1-13. Recuperado de: http://www.revistarazonypalabra.org/inde x.php/ryp/article/view/457

Bokser, J., y Salas-Porras, A. (1999). Globalización, identidades colectivas y ciudadanía. Política y Cultura, (12), 25-52.

Cancelo, M. (2007). La comunicación gubernamental. Revista Latinoamericana de Comunicación Chasqui, (98), 72-75. Recuperado de: http://chasqui.ciespal.org/index.php/chasqui /article/view/398/398

Castells, M. (2012). Redes de indignación y esperanza. Madrid, España: Alianza.

Colombo, G. (2012). Democracia. Buenos Aires, Argentina: Adriana Hidalgo Editora.

Delgado-Salazar, R. (2011). Educación para la ciudadanía desde la acción colectiva. Magis. Revista Internacional de Investigación en Educación, 4(7), 201-210.

Del Valle Rojas., C. (2007). Comunicación participativa: Aproximaciones desde América Latina. Revista Redes, (4), 113-130.
Elizalde, L., y Riorda, M. (2013) Comunicación gubernamental 360. Argentina: La Crujía Ediciones.

Hamlichi, B. (2008). Actores colectivos en Marruecos. THEOMAI (17), 89-97.

Involve. (2011). People \& Participation. How to put citizens at the heart of decisión-making. Recuperado de:

http://www.involve.org.uk/wp-content/upl oads/2011/03/People-and-Participation.pdf

Miller Moya, L. M. (2004). Acción colectiva y modelos de racionalidad. Estudios Fronterizos, 5(9), 107-130.

Molina, E., Curro, M. G., y Carrillo, M. (Marzo de 2016). Los modelos de comunicación gubernamental en materia ambiental. En La Agenda Emergente de las Ciencias Sociales: Conocimiento, Crítica e Intervención. Simposio llevado a cabo en el $5^{\circ}$ Congreso Nacional de Ciencias Sociales, Guadalajara, México.

Richter-Morales, U. (2014). De la protesta a la participación ciudadana. Ciudad de México: Océano.

Riorda, M. (2011). Hacia un modelo de comunicación gubernamental para el consenso. Ciencia Red. Recuperado de: http://www.cienciared.com.ar/ra/usr/9/257/fi sec04riorda.pdf

Rodrigo, M. (2014). Modelos de comunicación. Portal de la Comunicación InCom-UAB: El portal de los estudios de comunicación. Recuperado de: http://portalcomunicacion.com/u ploads/pdf/20_esp.pdf

Ruiz, J.I. (2012). Metodología de la investigación cualitativa. $5^{\text {a }}$. Edición. Bilbao, España: Editorial Deusto.

Villaveces-Niño, J. (2009). Acción colectiva y el proceso de la política pública. Ópera (9), $7-22$. 